\title{
Genetische Analyse des Legemusters in der Bodenhaltung im Hinblick auf neue Leistungs- prüfungskriterien für die Legehennenzucht
}

\author{
WIEBKE ICKEN ${ }^{1}$, STEFAN THURNER ${ }^{2}$, DAVID CAVERO ${ }^{1}$, MATTHIAS SCHMUTZ ${ }^{1}$, GEORG \\ WENDL $^{2}$ und RUDOLF PREISINGER ${ }^{1}$
}

\begin{abstract}
${ }^{1}$ Lohmann Tierzucht GmbH, Cuxhaven, Deutschland, ${ }^{2}$ Institut für Landtechnik und Tierhaltung der Bayerischen Landesanstalt für Landwirtschaft, Freising-Weihenstephan, Deutschland
\end{abstract}

\section{Zusammenfassung}

Zur gezielten Selektion von Legehennen für alternative Haltungssysteme ist eine Prüfung der individuellen Henne in der Umwelt, in der sie später gehalten wird als positiv zu bewerten. Eine neue technische Entwicklung, das "Weihenstephaner Muldennest", macht die automatische Erfassung der hierfür notwendigen Leistungs- und Nestverhaltensparameter des Einzeltieres in einem Gruppenhaltungssystem möglich. Von 263 Hennen der Linie Lohmann Silver wurden die exakten Eiablagezeitpunkte ermittelt und zur Analyse des Legemusters herangezogen. Einige Ergebnisse, die bereits in der Literatur herausgestellt wurden konnten auf diesem Weg bestätigt werden, andere brachten neue Erkenntnisse in Hinblick auf den Eiablagezeitpunkt und die Pausendauer im Anschluss an eine Legesequenz. Eine positive genetische Korrelation zwischen der Legesequenzlänge und der darauffolgenden Pausendauer $\left(r_{g}=+0,49\right)$ bestätigte die tendenziell etwas längeren Pausen im Anschluss an eine längere Legesequenz. Die Dauer einer Pause war allerdings ein weniger entscheidendes Kriterium für die Produktivität der Henne. Wichtiger war in diesem Zusammenhang die Anzahl der Pausen, die stetig mit der Anzahl der Legesequenzen über den gesamten Beobachtungszeitraum stieg. Legten die Hennen in kürzeren Sequenzen, so konnten anteilsmäßig mehr Sequenzen beobachtet werden, die sich in einer geringeren Anzahl registrierter Eier pro Henne widerspiegelte $\left(r_{g}=-0,73\right)$.

Eine logische Schlussfolgerung hieraus war die hohe positive Korrelation zwischen der Legesequenzlänge und der Anzahl registrierter Eier $\left(r_{g}=+0,82\right)$. Eng verbunden mit der Legesequenzlänge sowie der Legeleistung war der Parameter Zeitintervall zwischen zwei gelegten Eiern. Das Zeitintervall zwischen zwei gelegten Eiern reduzierte sich vom ersten bis zum dritten Ei einer Legesequenz stark und pendelte sich dann für die Mehrheit der Hennen auf etwas über 24 Stunden ein. Die phänotypische und genetische Beziehung dieses Zeitintervalls zur Legeleistung war negativ $\left(r_{p}=-0,46\right.$ und $\left.r_{g}=-0,46\right)$, die Heritabilität des Zeitintervalls wurde mit $h^{2}=0,17$ geschätzt und bewegte sich damit in der gleichen Größenordnung wie die Heritabilität für das Merkmal Eizahl $\left(h^{2}=0,20\right)$. Etwas höhere Erblichkeiten wurden für die indirekten Parameter Zeitintervall und Eiablagezeitpunkt des dritten bis fünften Eies einer Legesequenz geschätzt $\left(h^{2}=0,25, h^{2}=0,34\right.$ und $\left.h^{2}=0,29\right)$. Schätzwerte in diesem Bereich wären im Hinblick auf neue Leistungsprüfungsmerkmale durchaus konkurrenzfähig zu dem direkten Parameter Legeleistung und sollten deshalb in weiteren Untersuchungen getestet werden.

Schlüsselwörter: Legehenne, Legesequenzen, Zeitintervall, Legepausen, Eiablagezeitpunkt 


\section{Abstract}

\section{Genetic analysis of the laying pattern in floor management in terms of new performance parameters for breeding}

In order to facilitate a specific selection of laying hens which are suitable for alternative housing systems, it will be of advantage if the individual hens were tested in their later husbandry environment. Therefore, a new technology has been developed, the "Weihenstephan Funnel Nest Box", which enables the automatic recording of these essential performance and nesting behaviour parameters of the individual hens in a group housing system. The exact oviposition times of 263 laying hens of Lohmann Silver line were recorded and used to analyse the laying pattern. Some results which were already pointed out in literature were thus confirmed, other brought new cognitions in terms of the oviposition pattern and about the pause following a laying sequence. $A$ positive genetic correlation between the length of a laying sequence and the subsequent pause durations $\left(r_{g}=+0.49\right)$ confirmed the tendency to longer breaks following an increasing laying sequence. The duration of a break was, however, not such an essential criteria for the productivity of a hen. More important in this context was the number of pauses which rises constantly with the number of laying sequences over the whole observation period. When the hens laid in shorter sequences, it was observed that the proportion of sequences grew, which was reflected as smaller number of registered eggs per hen $\left(r_{g}=-0.73\right)$. The logical conclusion was the highly positive correlation between the length of a laying sequence and the number of registered eggs $\left(r_{g}=+0.82\right)$. Closely associated with the length of a laying sequence, as well as the laying performance, was the parameter time interval between two laid eggs. The time interval between two laid eggs was significant shortened from the first to the third egg within one laying sequence and eventually reached for more than $24 \mathrm{~h}$ for most of the hens. The phenotypic and genetic correlation between the time interval and the laying performance was negative $\left(r_{p}=-0.46\right.$ and $\left.r_{g}=-0.46\right)$. The heritability for the time interval was estimated at $h^{2}=0.17$ which was similar to the heritability for number of eggs $\left(h^{2}=0.20\right)$. Slightly higher heritabilities were estimated for the indirect parameter of the time interval, the oviposition time of the third to the fifth egg in a laying sequence $\left(h^{2}=0.25, h^{2}=0.34\right.$ and $\left.h^{2}=0.29\right)$. Valuations in this range would be in regard of new performance testing parameter competitive to the parameter number of eggs and therefore, be tested in further investigations.

Keywords: layers, laying sequence, time interval, oviposition time, genetic parameters

\section{Einleitung}

Die Merkmale zur Beschreibung des Legemusters einer Henne sind vielfältig und wie bereits aus der Literatur bekannt, nicht ganz unbedeutend, wenn z. B. nach züchterischen Möglichkeiten zur Verbesserung der Legeleistung gesucht wird. McCLUNG et al. (1976), YOO et al. (1988) und LILLPERS und WILHELMSON (1993) erfassten in konventionellen Einzelkäfigen unter anderem das Merkmal Zeitintervall zwischen zwei gelegten Eiern 
einer Legesequenz und berechneten hierfür genetische Parameter. Die geschätzten Heritabilitäten lagen im hoch erblichen Bereich. Die Korrelationen zeigten eine enge Beziehung zur Legeleistung sowie zu weiteren, das Legemuster einer Henne beschreibenden Merkmalen. Schon zu Beginn des 20. Jahrhunderts stellte ATWOOD (1929) heraus, dass kürzere Zeitintervalle mit längeren Legesequenzen verbunden sind und stellvertretend für eine produktivere Henne stehen. Diese Erkenntnis nutzten auch LUC et al. (1996). Sie untersuchten zwei Gruppen von Hennen, die auf ein hohes bzw. niedriges Eigelb-Eiweiß-Verhältnis selektiert wurden, hinsichtlich ihres Legemusters und stellten für beide Gruppen hohe Heritabilitäten für das Zeitintervall und die Anzahl der Legesequenzen sowie deren Länge heraus. Enge genetische Korrelationen der zwei hoch erblichen Merkmale, Anzahl Legesequenzen und Legesequenzlänge, zum Parameter Gesamteizahl wurden auch von BEDNARCZYK et al. (2000) ermittelt und daraus resultierend als erfolgversprechende Parameter im Selektionsindex von Legehennen beschrieben. Zu bedenken geben LILLPERS und WILHELMSON (1993) allerdings, dass besonders bei der Erfassung der Merkmale, Anzahl Legesequenzen und Legesequenzlänge, durch ein nicht registriertes Ei das Datenmaterial stark verzerrt wird. Einen deutlich geringeren Einfluss hätte ein derartiger Fehler in der Datenerfassung auf das jeweilige Zeitintervall und wäre deshalb aus dieser Sicht das bevorzugte Merkmal.

Ziel dieser Studie ist die statistisch genetische Analyse der verschiedenen Parameter einer Legeserie in der Bodenhaltung mit besonderem Augenmerk auf mögliche neue Selektionskriterien im Hinblick auf die züchterische Beeinflussung der Legehenne.

\section{Material und Methoden}

Für die Auswertungen bezüglich der Legesequenzen wurden 120026 Nestdaten an 350 auswertbaren Tagen (Januar bis Dezember 2005) von 263 durchgehend registrierten Legehennen der Herkunft Lohmann Silver ausgewertet. Die Hennen schlüpften im August 2004 und wurden in einem Stallabteil der Lehr- und Versuchsstation Thalhausen der Technischen Universität München aufgezogen. Im Alter von 17 Wochen wurden die Hennen in ein anderes Stallabteil, welches neben einer Voliere zusätzlich mit 48 Weihenstephaner Muldennestern (THURNER et al. 2005) ausgestattet war, umgestallt. Die Besonderheit dieser in zwei gleich langen Reihen, übereinander angeordneten Weihenstephaner Muldennester lag in ihrer technischen Ausstattung. Die Hennen wurden mit Hilfe der am Eingang des Nestes angebrachten Fanggabeln beim Betreten des Nestes vereinzelt und hatten jederzeit, ohne jegliche Fremdeinwirkung von außerhalb, die Möglichkeit das Nest wieder zu verlassen. Die Identifikation des einzelnen Tieres erfolgte mit Hilfe eines RFID Systems. Glastransponder (23 mm, HDX, Texas Instruments, ISO 11784/11785), wurden hierzu mit Hilfe eines Fußringes (RoxanID, LegBand) am Ständer jeder Henne befestigt. Im Nest wurde der Transponder von einer im Muldenboden integrierten trapezförmigen Antenne gelesen und die Daten mit der entsprechenden Uhrzeit registriert. Neben den Nestaufenthaltsdaten wurde auf diese Weise der exakte Eiablagezeitpunkt der Hennen mit Hilfe eines Sensors automatisch erfasst und die Ermittlung der einzelnen Legesequenzen ermöglicht. Vermehrte Unterbrechungen in den Legesequenzen traten mit Beginn des Monates März auf, da die Herde zu dieser Zeit von dem Bakterium Mannheimia haemolytica befallen war. 
Für die statistischen Analysen stand das Programmpaket SAS (SAS 2004), für die genetischen Untersuchungen VCE 4 (GROENEVELD 1998) zur Verfügung. Die Schätzung der Varianzkomponenten für die verschiedenen beschreibenden Merkmale einer Legesequenz erfolgte mit Hilfe der Restricted-Maximum-Likelihood-Methode (REML) unter Verwendung der Modelle 1 und 2.

Modell 1 (für das Merkmal Eiablagezeitpunkt):

$$
Y_{i j k}=\mu+p e_{i}+a_{j}+e_{i j k}
$$

wobei $Y_{i j k}$ der individuelle Beobachtungswert für das jeweilige Merkmal, separat für das 1. bis 5 . Ei innerhalb einer Legesequenz, $\mu$ der Mittelwert über alle Beobachtungen, $p e_{i}$ der permanente Umwelteffekt der Henne ${ }_{i}, a_{j}$ der additiv genetische Effekt des Tieres und $e_{i j k}$ der zufällige Resteffekt ist.

Modell 2 (für die Merkmale Legeleistung und Zeitintervall):

$$
Y_{i j}=\mu+a_{i}+e_{i j}
$$

wobei $Y_{i j}$ der individueller Beobachtungswert für das jeweilige Merkmal je Tier ${ }_{i}, \mu$ der Mittelwert über alle Beobachtungen, $a_{i}$ der additiv genetische Effekt des Tieres ${ }_{i}$ und $e_{i j}$ der zufällige Resteffekt ist.

\section{Ergebnisse}

Die Anzahl der Gelege wurde in Prozent für die jeweilige Größenklasse in Tabelle 1 dargestellt. Mit zunehmender Größe nahm die Häufigkeit der Gelege ab. Ab einer Größe von mehr als drei Eiern an aufeinanderfolgenden Tagen, wurden die einzelnen Gelege in Klassen zusammengefasst. Innerhalb dieser Klassen wurde ebenfalls eine abnehmende Tendenz mit steigender Anzahl Eier je Gelege beobachtet.

Tabelle 1

Prozentualer Anteil Gelege je Größenklasse

Percental share of clutches for each clutch size

\begin{tabular}{lc}
\hline Gelegegröße (Eier) & Anzahl, $\%$ \\
\hline 1 & 21,9 \\
2 & 11,8 \\
3 bis 5 & 19,3 \\
6 bis 10 & 15,8 \\
11 bis 20 & 14,1 \\
21 bis 49 & 12,5 \\
50 bis 99 & 3,7 \\
$>100$ & 0,8 \\
\hline
\end{tabular}

Übersteigt ein Gelege die Größenordnung von zwei Eiern, welches in dieser Studie in $78,1 \%$ der Fälle zutraf, so wird von einer Legesequenz gesprochen. Die maximal erreichte Legesequenzlänge betrug 150 Eier, welche maßgeblich die durchschnittliche Legesequenzlänge und die Anzahl der Legesequenzen je Henne über den gesamten Beobachtungszeitraum beeinflusste. Für die meisten Hennen galt: Je kürzer die maximale Legesequenz, desto größer die Anzahl der beobachteten Legesequenzen und desto geringer die mittlere Anzahl gelegter Eier innerhalb einer Sequenz (Abbildung 1). 


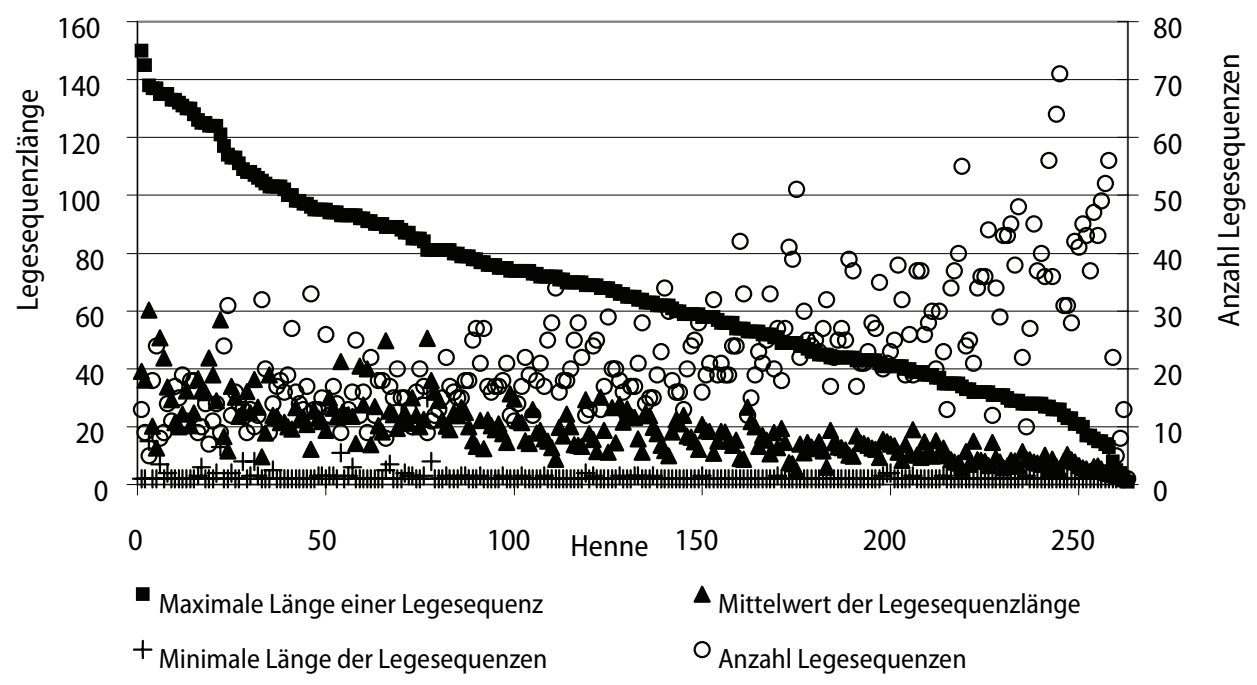

Abbildung 1

Anzahl Legesequenzen je Henne, sowie deren durchschnittliche, minimale und maximale Legesequenzlänge, geordnet nach der maximalen Legesequenzlänge

Number of sequences per hen, as well as the mean, minimum and maximum length of the laying sequence, sorted by the maximal sequence length)

Das stotternde Legen von einem $\mathrm{Ei}$, sowie die sehr kurzen Legesequenzen wurden hauptsächlich in den ersten beiden Legemonaten und ganz am Ende der Legezeit vermerkt. Sie reduzierten die mittlere Legesequenzlänge um 3,4 Eier auf 11,7 Eier. An jede Legesequenz schließt sich eine Legepause an, die in $63 \%$ aller Fälle einen Tag lang war. Längere Legepausen von mehr als 5 Tagen wurden bei dieser Herde fast ausschließlich während des Krankheitseinbruches im März bemerkt. Eine Einteilung der Hennen nach ihrer maximal erreichten Legesequenzlänge in drei Gruppen (Tabelle 2) dient der Beschreibung des Legemusters von Hennen unterschiedlicher Produktivität.

\section{Tabelle 2}

Durchschnittliche Anzahl Legesequenzen und deren Legesequenzlänge, sowie des prozentualen Anteils an 1-, 2-, 3- und längeren Tages-Pausen je Hennengruppe

Mean number of laying sequences and their length, as well as the fraction percentage of 1, 2, 3 and longer pause days per hen group

\begin{tabular}{lccc}
\hline Gruppe (max. Legesequenzlänge) & $1(>=100$ Eier $)$ & $2(>=50$ und $<100$ Eier $)$ & $3(<50$ Eier $)$ \\
\hline N [Hennen] & 41 & 126 & 78 \\
mittlere Anzahl Legesequenzen & 15 & 19 & 32 \\
mittlere Legesequenzlänge & 29 & 21 & 11 \\
Anteil 1 Tages-Pausen, \% & 64 & 65 & 61 \\
Anteil 2 Tages-Pausen, \% & 16 & 15 & 20 \\
Anteil 3 Tages-Pausen, \% & 6 & 7 & 7 \\
Anteil 4 Tages-Pausen und länger & 14 & 13 & 12 \\
Legeleistung, \% & 83 & 80 & 70 \\
\hline
\end{tabular}

Der Kategorie 2 ( $>=50$ Eier $<100)$ konnten die meisten Hennen zugeordnet werden. Sie lagen bezüglich der Anzahl Legesequenzen und der Legesequenzlänge zwischen den Hennen der Gruppen 1 und 3. Die längsten Legesequenzen mit durchschnittlich 29 Eiern 
an aufeinanderfolgenden Tagen, sowie die geringste Anzahl an Legeserien, hatten die Tiere aus Gruppe 1. Der Anteil der 1-Tages-Pausen überwog mit mehr als $60 \%$ deutlich in allen Gruppen. Der Anteil an 2-Tages-Pausen variierte von 15 bis 20\%, der der 3-TagesPausen war mit 6 bzw. 7\% relativ konstant. Im Vergleich der Gruppen erreichten die Tiere der 2. Gruppe den höchsten Prozentsatz an 1-Tages-Pausen, gefolgt von den Hennen der 1. und 3. Gruppe. Die Hennen mit der kürzesten maximalen Legesequenz legten die wenigsten Eier. Ihre geringe Produktivität ist vor allem auf die hohe Anzahl kurzer Legepausen, die stetig mit der Häufigkeit der Legeserien steigt, zurückzuführen. Die Dauer der einzelnen Pause scheint nur eine geringe Bedeutung im Hinblick auf die Produktivität der Henne zu haben, allerdings wird eine leichte Beeinflussung von Seiten der Henne durch die Länge der vorhergehenden Legesequenz vermutet.

Im Hinblick auf eine Verschiebung der Eiablagezeiten im Tagesverlauf innerhalb der Legesequenzen wurde das jeweils erste und letzte Ei einer Serie analysiert. Die Eiablage des zuerst gelegten Eies über alle Serien erfolgte vornehmlich in den Morgenstunden, verstärkt zwischen 6:30 Uhr und 6:45 Uhr.

Die Verteilung des Eiablagezeitpunktes des jeweils letzten Eies verlief hingegen dreigipfelig. In der Zeit von 6:15 Uhr bis 6:45 Uhr, von 8:00 Uhr bis 8:30 Uhr und von 13:30 Uhr bis 14:00 Uhr waren Maximalwerte in der Verlaufskurve des letzten Eies einer Sequenz zu beobachten (Abbildung 2). Die gleiche Auswertung in Abhängigkeit von der Legesequenzlänge ergab mit zunehmender Serienlänge tendenziell eine breitere Verteilung der Eiablagezeiten des ersten Eies, wohingegen der Verlauf für das letzte Ei einer Legesequenz annähernd gleich blieb. Eine derartige Häufigkeitsverteilung der Eiablagezeitpunkte lässt vermuten, dass die Zeitdifferenz zwischen zwei gelegten Eiern einer Sequenz in den meisten Fällen etwas mehr als 24 Stunden betrug, und sich in längeren Legesequenzen immer mehr dem 24-Stunden-Rhythmus annäherte.

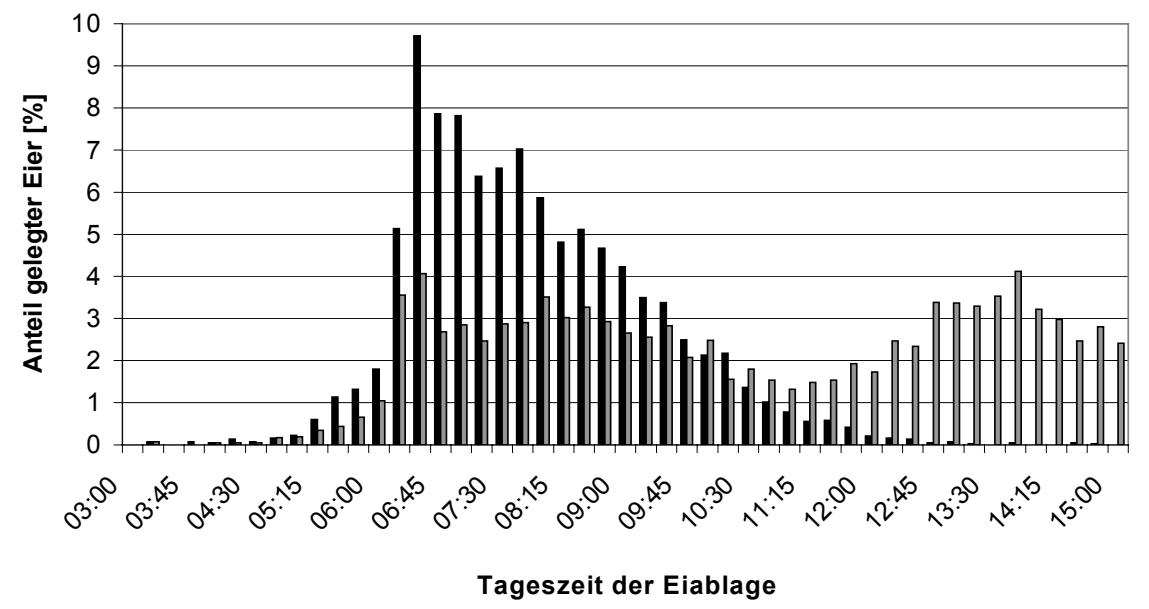

घ erstes Ei einer Legesequenz $\square$ letztes Ei einer Legesequenz

Abbildung 2

Prozentuale Verteilung des Eiablagezeitpunktes des ersten und letzten Eies einer Legesequenz Percental allocation of the oviposition time of the first and last egg in a laying sequence 
Eine genauere Beschreibung des Eiablageintervalls innerhalb der Legesequenzen war anhand von Abbildung 3 möglich. In dieser Grafik wurde das Zeitintervall zum vorherigen Ei einer Legesequenz dargestellt. Die Skalierung auf der X-Achse beginnt mit dem zweiten Ei einer Serie, da vor dem ersten Ei eine Pause gemacht wurde und der Zeitabschnitt über 36 Stunden lag. Alle Hennen reduzierten ihr Zeitintervall vom ersten bis zum dritten Ei stark und pendelten sich dann mit leicht sinkender Tendenz auf einem Niveau von etwas über 24 Stunden ein. Die Hennen der 3. Gruppe benötigten vom ersten bis zum letzten Ei durchschnittlich etwas mehr Zeit als die Hennen mit längeren Sequenzen. Am Ende langer Sequenzen der Hennen aus Gruppe 1 nimmt die Variation der Zeitintervalle zu. Die Abbildung 3 verdeutlichte aber auch, dass Zeitintervalle von weniger als 24 Stunden möglich sind. Legehennen, die solch kurze Produktionszeiten aufwiesen, rutschten mit der Eiablage im Tagesverlauf keineswegs nach hinten, sie produzierten ihr folgendes Ei sogar früher am Tag. Andere Hennen, zu denen der Großteil der Herde zählt, verlagern ihren Eiablagezeitpunkt mit einem Zeitintervall von über 24 Stunden nach hinten und könnten so bei langen Legesequenzen über die Nestöffnungszeiten hinausragen mit der Bedingung einen Tag aussetzen zu müssen.

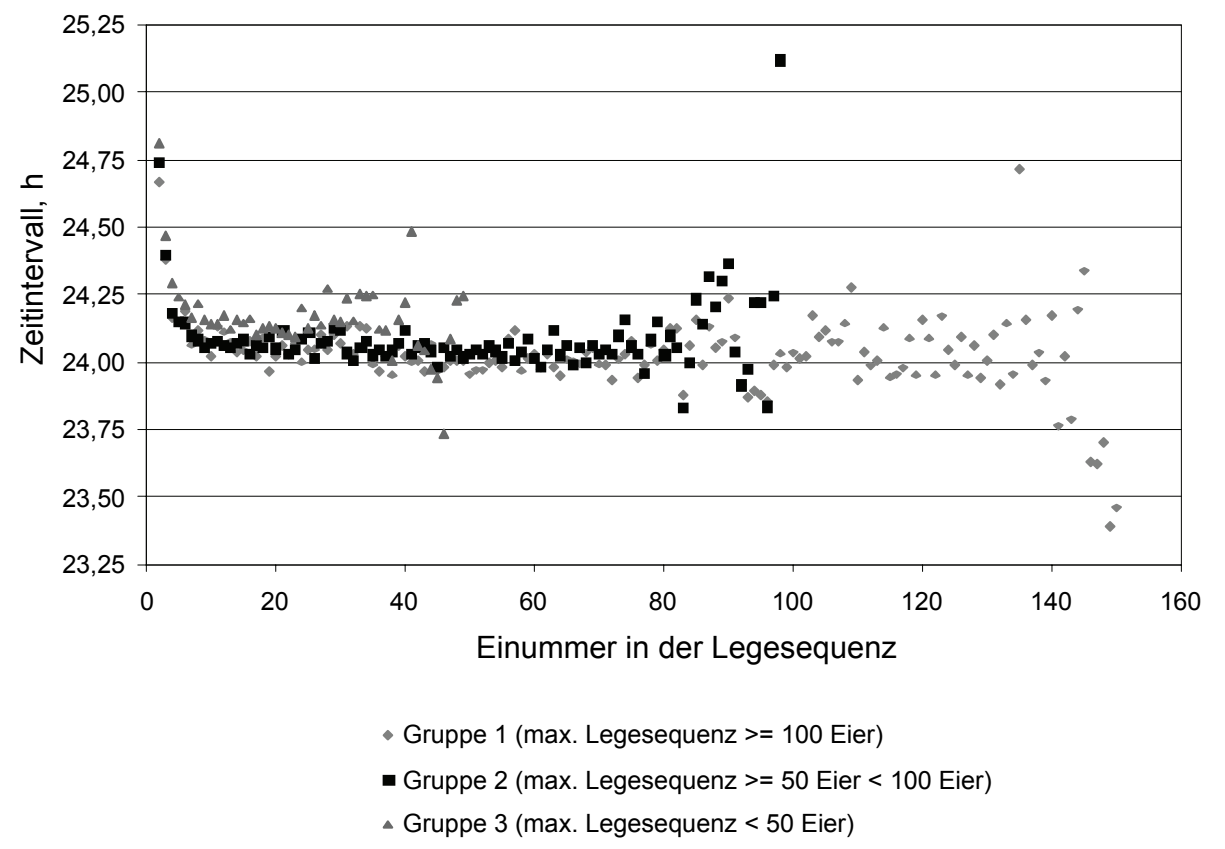

Abbildung 3

Das Zeitintervall zwischen zwei aufeinanderfolgenden Eiern innerhalb einer Legesequenz in Abhängigkeit von der maximalen Legesequenzlänge einer Henne (Gruppeneinteilung).

The time interval between two following eggs in a laying sequence, depending on the maximal length of the laying sequence of each hen

In Abbildung 4 wurde der Anteil gelegter Eier je Zeitintervall, unabhängig von der Einummer in einer Legesequenz, dargestellt. Der Legerhythmus der meisten Hennen lag ähnlich wie in Abbildung 4 herausgestellt, zwischen 24 Stunden und 24 Stunden und 30 
Minuten. Mehr als $1 / 4$ aller Eier wurden aber auch in weniger als 24 Stunden von den Hennen gelegt. Die Gruppeneinteilung, nach der jeweils maximalen Legesequenzlänge einer Henne, verdeutlichte, dass die Eier mit einer Produktionszeit von weniger als 24 Stunden vermehrt von denjenigen Hennen gelegt wurden, die über Wochen, ohne Pause, jeden Tag ein Ei gelegt haben. Die Hennen der Gruppe 3 produzierten den größten Anteil der Eier, die in einem Zeitintervall von 26 bis 36 Stunden gelegt wurden. Die Eier, bei denen mehr als 36 Stunden zum vorherigen Ei derselben Henne vergangen war, wurden nach einer Legepause von mindestens einem Tag gelegt. Die Legesequenz wurde in diesen Fällen unterbrochen. Wie erwartet fielen auch in diesen Bereich hauptsächlich Eier von Hennen mit einer kürzeren maximalen Legesequenz.

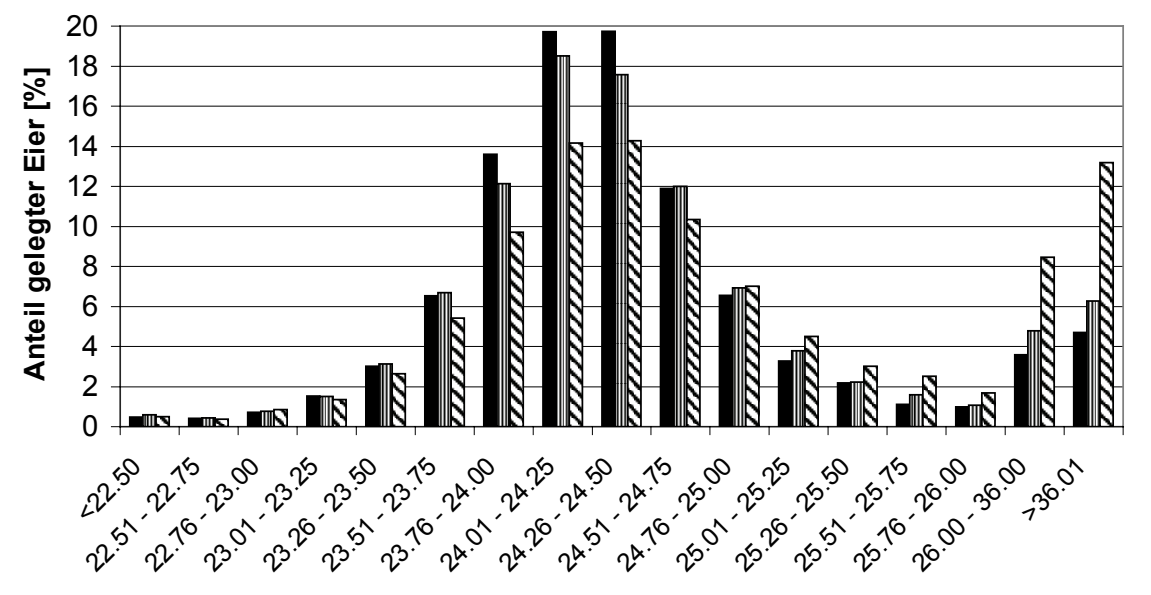

Zeitintervall zwischen zwei gelegten Eiern [h]

- Gruppe 1 ( $\max$. Legesequenz >= 100 Eier)

四 Gruppe 2 (max. Legesequenz >= 50 Eier $<100$ Eier)

$₫$ Gruppe 3 (max. Legesequenz $<50$ Eier)

\section{Abbildung 4}

Prozentualer Anteil gelegter Eier je Zeitintervall zwischen zwei Eiern einer Legesequenz

Percental number of laid eggs per time interval between two eggs of one laying sequence

Die Zeitpunkte der Eiablage des ersten bis fünften Eies einer Legesequenz waren eng miteinander verbunden. Die phänotypischen Korrelationen für diese Merkmale sind in Tabelle 3 dargestellt.

Tabelle 3

Phänotypische Korrelationen zwischen den Eiablagezeiten für das jeweilige Ei einer Legesequenz (LS) Phenotypic correlations between the oviposition times for the respective egg in a laying sequence (LS)

\begin{tabular}{lcccc}
\hline & 2. Ei einer LS & 3. Ei einer LS & 4. Ei einer LS & 5. Ei einer LS \\
\hline 1. Ei einer Legesequenz & $+0,84$ & $+0,68$ & $+0,55$ & $+0,55$ \\
2. Ei einer Legesequenz & & $+0,92$ & $+0,82$ & $+0,68$ \\
3. Ei einer Legesequenz & & & $+0,94$ & $+0,83$ \\
4. Ei einer Legesequenz & & & & $+0,92$ \\
\hline
\end{tabular}


Die positiven Beziehungen zueinander waren zwischen unmittelbar aufeinanderfolgenden Eiern einer Sequenz am größten und erreichten Werte von $r_{p}=+0,94$. Mit zunehmender Entfernung sanken die positiven Korrelationen auf ein mittleres Niveau. Der Eiablagezeitpunkt eines Eies scheint demnach stark von dem des zuvor gelegten Eies abhängig zu sein.

Eine leichte Verschiebung der durchschnittlichen Eiablagezeiten vom ersten zum fünften Ei einer Legeserie zeigen die in Tabelle 4 aufgelisteten Mittelwerte. Die geschätzten Heritabilitäten für den Eiablagezeitpunkt des ersten bis fünften Eies einer Legesequenz lagen im Bereich von $\mathrm{h}^{2}=0,05$ bis $\mathrm{h}^{2}=0,34$, mit niedrigen Standardfehlern. Neben den Eiablagezeitpunkten aufeinanderfolgender Eier wurde der Zusammenhang zwischen dem Eiablagezeitpunkt des letzten Eies einer Legesequenz und des ersten Eies der neuen Sequenz geprüft sowie deren Beziehung zur Legesequenzlänge und Pausendauer. Eine positive genetische Korrelation bestand zwischen der Länge der Sequenz und der folgenden Pausendauer $r_{g}=+0,49$. Demnach machten die Hennen im Anschluss an eine größere Legeserie eine längere Pause. Weiterhin wurde eine positive phänotypische Beziehung zwischen der Länge der letzten und folgenden Legesequenz geschätzt $\left(r_{p}=+0,28\right)$. Eine Beeinflussung der Legeleistung durch das Legesequenzmuster konnte mit Hilfe entsprechender Korrelationsberechnungen nachgewiesen werden. Die Anzahl gelegter Eier je Henne stieg mit zunehmender mittlerer Legesequenzlänge $\left(r_{g}=+0,82\right)$, sowie mit einer abnehmenden Anzahl Legesequenzen über den Untersuchungszeitraum $\left(r_{g}=-0,73\right)$.

Tabelle 4

Mittelwert ( $\bar{x})$ und Standardabweichung (std), sowie die Heritabilität $\left(\mathrm{h}^{2}\right)$ und deren Standardfehler (SE) für den Eiablagezeitpunkt des ersten bis fünften Eies einer Legesequenz

Mean $(\bar{x})$ and standard deviation (std), as well as the heritability $\left(h^{2}\right)$ and their standard error (SE) for the oviposition time of the first to the fifth egg in a laying sequence

\begin{tabular}{lcccc}
\hline & $\overline{\mathrm{x}}, \mathrm{h}: \mathrm{mm}$ & $\mathrm{std}, \mathrm{h}: \mathrm{mm}$ & $\mathrm{h}^{2}$ & $\mathrm{SE}$ \\
\hline 1. Ei einer Legesequenz & $7: 45$ & $1: 23$ & 0,05 & 0,03 \\
2. Ei einer Legesequenz & $8: 10$ & $1: 17$ & 0,16 & 0,06 \\
3. Ei einer Legesequenz & $8: 22$ & $1: 21$ & 0,25 & 0,08 \\
4. Ei einer Legesequenz & $8: 28$ & $1: 26$ & 0,34 & 0,10 \\
5. Ei einer Legesequenz & $8: 38$ & $1: 41$ & 0,29 & 0,10 \\
\hline
\end{tabular}

Die Ergebnisse der Varianzanalyse, basierend auf dem Modell 2, für das mittlere Zeitintervall zwischen zwei gelegten Eiern einer Legesequenz je Henne und deren jeweiliger Eizahl (gesamt) enthält Tabelle 5. Auffällig sind die identischen negativen Korrelationswerte $\left(r_{g}\right.$ und $\left.r_{p}\right)$ zwischen den beschriebenen Parametern $\left(r_{g}=-0,46\right)$. Ein kürzeres Zeitintervall stünde damit stellvertretend für eine höhere Legeleistung.

Tabelle 5

Heritabilitäten (Diagonale), genetische (oberhalb der Diagonale) und phänotypische (unterhalb der Diagonale) Korrelation zwischen den Merkmalen mittlere Legeleistung je Henne und mittlerem Zeitintervall zwischen zwei Eiern einer Legesequenz

Heritabilities (diagonal), the genetic (upper diagonal) and phenotypic (lower diagonal) correlation between the parameters mean laying performance for each hen and mean time interval between two eggs in a laying sequence

\begin{tabular}{lcc}
\hline & Legeleistung & Zeitintervall \\
\hline Legeleistung & 0,20 & $-0,46$ \\
Zeitintervall & $-0,46$ & 0,17 \\
\hline
\end{tabular}




\section{Diskussion}

In der Vergangenheit wurden bereits Untersuchungen zu den Legesequenzen einzelner Hennen, in herkömmlichen Einzelkäfigen (McCLUNG et al. 1976, AYORINDE und OLAGBUYIRO 1991, LILLPERS und WILHELMSON 1993, LUC et al. 1996, BEDNARCZYK 2000, AKBAS et al. 2002, CHEN und TIXIER-BOICHARD 2003) und mit Hilfe der Fallnestkontrolle (ATWOOD 1929, HEYWANG 1938) durchgeführt. In verschiedenen Herden unterschiedlichster Versuchsreihen wurden ähnliche Verteilungsmuster für die durchschnittliche Legesequenzlänge ermittelt. Die am häufigsten gemessenen Legesequenzen waren die mit einer Länge von zwei und drei Eiern. ATWOOD (1929) und HEYWANG (1938) ermittelten für $45 \%$ der Gelege eine Größe von zwei Eiern. Einzeln gelegte Eier, bei denen die Henne sowohl an dem vorangegangenen Tag, als auch an dem darauffolgenden Tag kein Ei gelegt hat traten vorwiegend zu Beginn des Untersuchungszeitraumes auf. BROERSKY (1984) schätzte ihren Anteil auf 16\%, AYORINDE und OLAGBUYIRO (1991) auf 32\%. Der in diesem Versuch herausgestellte Anteil von Gelegen mit einer Größenordnung von einem Ei lag mit 22\% dazwischen (Tabelle 1). Die meisten Sequenzen (ca. 20\%) erstreckten sich bei BROERSKY (1984) über drei Eier. Überstieg das Gelege eine Größe von drei Eiern, so galt wie bei den anderen zitierten Autoren das Prinzip der abnehmenden Häufigkeit mit zunehmender Legesequenzlänge. Eine starke Abhängigkeit der Anzahl Legesequenzen mit einer Länge bis zu zehn Eiern, von der Dauer des Untersuchungszeitraumes, konnte anhand der variierenden Zeiträume (1 bis 14 Monate) in der zitierten Literatur, sowie durch eine Studie von BEDNARCZYK et al. (2000) ausgeschlossen werden. BEDNARCZYK et al. (2000) berechneten Durchschnittswerte für die Länge der Legesequenzen und deren Anzahl in zwei Untersuchungszeiträumen (bis zur 38. und bis zur 64. Lebenswoche) verschiedener Generationen mit 2789 bzw. 2210 Hennen der Rasse Rhode Island White. Die maximale Legesequenzlänge betrug für den ersten Zeitraum bis zur 38. Lebenswoche im Durchschnitt 31,4 bzw. 42,4 Tage und war damit wesentlich kürzer als für den zweiten Zeitraum (43,9 bzw. 47,8 Tage). Die durchschnittlichen Legesequenzlängen über alle Beobachtungen lagen hingegen in beiden Generationen für die zweite Periode niedriger als für die erste. Diese Entwicklung ist auf den überproportionalen Anstieg in der Anzahl Legesequenzen und die damit verbundene Reduzierung der Legesequenzlänge im zweiten Beobachtungsabschnitt, sowie auf das rückläufige Leistungsniveau mit zunehmendem Alter zurückzuführen. Die Untersuchungen des längeren Zeitraumes lassen demnach vermuten, dass die Hennen in der Lage sind in langen Sequenzen zu legen, diese hohe Leistung (mehrere lange Legesequenzen) aber nicht durchhalten können. Die mittlere Legesequenzlänge verkürzt sich demnach mit zunehmendem Alter der Hennen. Untersuchungen anderer Autoren, die den Einfluss des Alters der Tiere auf die Legesequenzen analysierten, bestätigten dies. BROERSKY (1984) zeigte vom ersten zum zweiten Legemonat einen deutlichen Anstieg der durchschnittlichen Legesequenzlänge auf bis zu zehn Eier, die in den anschließenden acht Legemonaten, mit zunehmenden Alter der Hennen, auf ein Minimum absank. Die Beziehung des mit der Legesequenzlänge eng verbundenen Zeitintervalls zwischen zwei gelegten Eiern zum Alter wurde schon früh von ATWOOD (1929) herausgestellt. Er schlussfolgerte, dass 
längere Legesequenzen kürzere Zeitintervalle zwischen zwei aufeinanderfolgenden Eiern aufweisen und diese Zeitabstände mit zunehmendem Alter größer werden. Bestätigt wurden diese Aussagen später von HEYWANG (1938), AMIN-BAKHCHE und MÉRAT (1975) und LILLPERS und WILHELMSON (1993). Die mittleren Zeitabstände zwischen zwei gelegten Eiern an aufeinanderfolgenden Tagen variieren nicht nur in Abhängigkeit von der Legesequenzlänge bzw. innerhalb der Legesequenzen (ATWOOD 1929, HEYWANG 1938) sondern auch von der Rasse (HEYWANG 1938), von Individuum zu Individuum (WARREN und SCOTT 1934), von der Tageslänge (YOO et al. 1986) und verschiedenen Selektionskriterien, wie der Eizahl, dem Eigewicht oder dem Eigelb-Eiweiß-Verhältnis (LILLPERS und WILHELMSON 1993, LUC et al. 1996). In Abbildung 3 wurden die Zeitintervalle in Abhängigkeit von der jeweiligen Einummer in einer Legesequenz für die drei verschiedenen Gruppen dargestellt. Der mittlere Zeitabstand sank unabhängig von der maximalen Legesequenzlänge eines Tieres (Gruppe 1-3) vom ersten zum dritten Ei stark und pendelte sich für alle weiteren Eier einer Sequenz, mit einigen Ausnahmen, auf einem Niveau zwischen 24 Stunden und 24,25 Stunden ein. Des Weiteren wurden Zeitintervalle von weniger als 24 Stunden ermittelt, von denen auch andere Autoren, wie z.B. YOO et al. (1986) berichteten. Das mittlere Zeitintervall variierte in ihren Untersuchungen in Abhängigkeit von der Tageslänge zwischen 23,1 und 27,6 Stunden bei 24 stündiger Lichtgabe und von 24,1 bis 26,8 Stunden unter »normalen Lichtbedingungen « (15,25 h Licht, 8,75 h Dunkelheit). Zeitabstände zwischen 24 und 28,1 Stunden ermittelten WARREN und SCOTT (1934) für einzelne Hennen. LILLPERS und WILHELMSON (1993) stellten die Intervalle für drei, auf verschiedene Merkmale selektierte Linien, heraus. Die Gruppe von Hennen, die auf eine hohe Eizahl selektiert wurden wiesen die kürzesten, die der auf Eimasse selektierten Gruppe die längsten Zeitspannen auf. Diese Verteilung lässt vermuten, dass die Anzahl gelegter Eier mit dem Zeitintervall negativ korreliert ist. Die in Tabelle 5 dargestellte Korrelation $\left(r_{g}=-0,46\right)$ bestätigt diese negative Beziehung, ebenso wie die Ergebnisse anderer Studien. YOO et al. (1988) ermittelten genetische und phänotypische Korrelationen in der Größenordnung $r_{g}=-0,73$ und $r_{p}=-0,36$ für das Zeitintervall zwischen zwei Eiern einer Legesequenz und der Legerate vom ersten Ei bis zur 65. Lebenswoche. Eine etwas schwächere Beziehung stellten MCCLUNG et al. (1976) heraus. In ihren Ergebnissen wurden die Korrelationen zwischen dem Zeitintervall und der Anzahl Eier bis zum 280. bzw. 500. Tag aufgeführt, $r_{g} 280=-0,45$ bzw. $r_{g} 500=+0,20$ und $r_{p 280}=-0,34$ bzw. $r_{p 500}=-0,06$. Eine deutliche Tendenz der negativen Korrelationen über den Beobachtungszeitraum von 280 Tagen hin zu positiven Werten für die Untersuchungen über 500 Tage war erkennbar. Als mögliche Einflussfaktoren auf die Entwicklung der Korrelationswerte wurden der Verlauf der Eiproduktionskurve und die Umwelt (Management, Fütterung, Krankheitskontrolle, Lichtprogramm) genannt. Die Eiproduktion stieg wie erwartet vom 174. bis 280 . Tag schnell und fiel anschließend langsam ab. Die in dieser Studie herausgestellten genetischen Korrelationen zwischen der Anzahl gelegter Eier und der mittleren Legesequenzlänge bzw. der Anzahl Legesequenzen $r_{g}=+0,82$ und $r_{g}=-0,73$ können ähnlich wie bei YOO et al. (1988) und McCLUNG et al. (1976), für den Beobachtungszeitraum von 200 Tagen, interpretiert werden, da die Legesequenzlänge negativ mit dem Zeitintervall korreliert ist (ATWOOD 1929) und die Anzahl der Legesequenzen mit 
abnehmender Legesequenzlänge steigt (BEDNARCZYK et al. 2000). Mittlere bis hoch negative Korrelationen zwischen der Anzahl gelegter Eier und der Anzahl an Legesequenzen wurden wie erwartet von BEDNARCZYK et al. (2000) ermittelt.

Die logische Überlegung die hinter dieser Beobachtung steht, ist die zunehmende Anzahl der Tage, an denen kein Ei gelegt wird (Pause), wenn die Hennen ihre Eier in mehreren kurzen Sequenzen legen. Die Pausendauer betrug in mehr als $60 \%$ aller Fälle, unabhängig von der maximalen Legesequenzlänge, lediglich einen Tag (Tabelle 2). Längere Pausen als 3 Tage, traten nur in einigen wenigen Situationen auf. Die herausgestellte positive genetische Beziehung zwischen der Länge der Legesequenz und der darauffolgenden Pausendauer $r_{g}=+0,49$ verdeutlichte, dass diese längeren Pausen im Anschluss an eine längere Legesequenz getätigt wurden. In der Studie von LUC et al. (1996) wurden die Mittelwerte für die Zeitverzögerung in der Eiablage nach einem Tag Pause angegeben, welche 17,5 Stunden betrug. Demnach beginnen die Hennen etwas früher am Tag mit der Ablage ihres ersten Eies, als sie in der vorigen Legesequenz mit dem letzten Ei geendet haben. In Abbildung 2 wurde eine ähnliche Tendenz aus den analysierten Daten dargestellt. Das erste Ei einer Legesequenz wird hauptsächlich am frühen Vormittag gelegt, während die Verteilung der Eiablagezeiten der letzten Eier über die gesamten Nestöffnungszeiten variierte. Die mittleren Eiablagezeiten vom ersten bis zum fünften Ei wurden in Tabelle 4 dargestellt und zeigten den größten Abstand vom ersten zum zweiten Ei, welches sich mit dem beschriebenen Zeitintervall in Abbildung 3 deckt. Die Verschiebung des mittleren Eiablagezeitpunktes vom zweiten zum dritten und dritten zum vierten Ei betrug hingegen immer nur die Hälfte des vorangegangenen Abstandes, bis sich die Hennen auf einem individuellen Niveau von mehrheitlich etwas über 24 Stunden einpendelten. Die genetische Veranlagung für die einzelnen Eier innerhalb einer Legesequenz wurde in Tabelle 4 mit Hilfe der Heritabilitäten dargestellt. Die geschätzten Erblichkeitswerte für den Eiablagezeitpunkt des ersten bis fünften Eies einer Serie lagen im niedrigen bis mittleren Bereich $\left(h^{2}=0,05\right.$ bis $\left.h^{2}=0,34\right)$, während die Heritabilität für das mittlere Zeitintervall aller Legesequenzen einer Henne einen Wert von $\mathrm{h}^{2}=0,17$ annahm und damit deutlich unter den geschätzten Heritabilitäten in der zitierten Literatur lag. McCLUNG et al. (1976) schätzten die Erblichkeiten mit verschiedenen Modellen und erzielten, basierend auf einem Vatermodell, einen Schätzwert für das Zeitintervall von $\mathrm{h}^{2}=0,33$. YOO et al. (1988) schätzten für das gleiche Merkmal, ebenfalls mit einem Vatermodell, höhere Heritabilitäten $\mathrm{h}^{2}=0,47$ unter »normalen Lichtbedingungen« $\left(15,25 \mathrm{~h}\right.$ Licht, $8,75 \mathrm{~h}$ Dunkelheit) und $\mathrm{h}^{2}=0,67$ bei ständiger Beleuchtung. Ebenfalls hoch erbliche Schätzwerte erzielten LILLPERS und WILHELMSON (1993) für das mittlere Zeitintervall zwischen zwei Eiern einer Legesequenz $\left(h^{2}=0,42\right.$ bis $\left.h^{2}=0,55\right)$.

Die herausgestellten genetischen Parameter für die verschiedenen Merkmale, die in Zusammenhang mit einer Legesequenz ermittelt werden können, lieferten interessante Ergebnisse für die weitere züchterische Bearbeitung der Legehenne. Der enge genetische Zusammenhang zwischen dem Zeitintervall zwei gelegter Eier einer Sequenz und der Legeleistung zeigten z.B., dass der Zeitabstand ein gutes Selektionskriterium im Hinblick auf den Leistungsparameter Eizahl liefert. Die geschätzte Heritabilität für den »neuen« Parameter Zeitintervall lag in diesem Versuch im niedrig erblichen Bereich, ähnlich wie die aus der Literatur bekannten Heritabilitätsschätzwerte für die Legeleistung, wurde 
aber von anderen Autoren als mittel bis hoch erbliches Merkmal eingeschätzt. Die anderen analysierten Merkmale einer Legesequenz, wie die Anzahl, die durchschnittliche Länge, die Pausendauer oder die Eiablagezeitpunkte des ersten bis fünften Eies einer Serie waren eng miteinander verzahnt und lieferten für die Beschreibung des Legemusters einer Henne ähnliche Resultate, wie der Parameter Zeitintervall. Weitere Varianzanalysen auch für diese Merkmale wären deshalb für weitere Linien und genetische Gruppen mit einem größeren Stichprobenumfang wünschenswert, um verschiedene Parameter im Hinblick auf ein späteres Leistungsprüfungskriterium zu validieren.

\section{Literatur}

Akbas Y, Ünver Y, Oguz I, Altan Ö (2002) Estimation of genetic parameters for clutch traits in laying hens 7th World Congress on Gen Appl Livest Prod August 19-23 Montpellier France

Amin-Bakhche M, Mérat $P$ (1975) Study of dwarf gene related to the sex of laying hens: Oviposition time and characteristics of following eggs in a laying sequence. Ann Génét Sél anim 7, 13-22 [in French]

Atwood H (1929) Observations concerning the time factor in egg production. Poultry Sci 8 137-40

Ayorinde KL, Olagbuyiro B (1991) The influence of clutch size and oviposition time on egg weight and egg index in hubbard layers. Bull Anim Health Prod Afr 39, 251-3

Bednarczyk M, Kiectzewski K, Swaczkowski T (2000) Genetic parameters of the traditional selection traits and some clutch traits in a commercial line of laying hens. Arch Geflügelk 64, 129-33

Broersky AV (1984) Lay recurrence and time parameters during reproductive period Sel'skochozjajstvennaja Biol 1184-88 [in Russian]

Chen CF, Tixier-Boichard M (2003) Estimation of genetic variability and selection response for clutch length in dwarf brown-egg layers carrying or not the naked neck gene. Gen Sel Evol 35, 219-38

Groeneveld E (1998) VCE4 User's Guide and Reference Manual. Version 1.3 November 1998

Heywang BW (1938) The time factor in egg production. Poultry Sci 17, 240-7

Lillpers K, Wilhelmson M (1993) Genetic and phenotypic parameters for oviposition pattern traits in three selection lines of laying hens. British Poultry Sci 34297-308

Luc KM, Miyoshi S, Suzuki M, Mitsumoto T (1996) Heritability estimates of some clutch traits in the laying hen. Jap Poultry Sci 33, 23-8

McClung MR, Wang ABS, Jones WT (1976) Response to selection for time interval between ovipositions in the hen. Poultry Sci 55, 160-71

SAS (2004) SAS/STAT User's Guide (Release 8) SAS Institute Cary NC USA

Thurner S, Böck S, Weinfurtner R, Fröhlich G, Wendl G (2005) Individual and automatic recording of laying performance and behaviour - Funnel Nest Box for laying hens in group housing systems. Landtechnik 60, 280-1 [in German]

Warren DC, Scott HM (1934) The time factor in egg formation. Poultry Sci 14, 195-207

Yoo BH, Sheldon BL, Podger RN (1986) Analyses of oviposition times and intervals in a wide range of layer flocks under normal and continous lighting regimes. Brit Poultry Sci 27, 267-88

Yoo BH, Sheldon BL, Podger RN (1988) Genetic parameters for oviposition time and interval in a White Leghorn population of recent commercial origin. Brit Poultry Sci 29627-37

Received 14 November 2007, accepted 22 June 2008.

Corresponding author:

WIEBKE ICKEN

email: icken@ltz.de

Lohmann Tierzucht GmbH, Am Seedeich 9-11, 27454 Cuxhaven, Germany 